\title{
An Easy Tuning Fuzzy Pulse Pump Controller for a Phase/Frequency Locked Loop Servomechanism
}

\author{
Liang-Rui Chen, Hahn-Ming Lee, and Guan-Chyun Hsieh \\ Department of Electronic Engineering \\ National Taiwan University of Science and Technology \\ Taipei, Taiwan 106, ROC
}

\begin{abstract}
Fuzzy set theory had been successfully used to develop a fuzzy pulse pump controller (FPPC) for a phase/frequency locked loop servomechanism (PFLS). For self-tuning the membership functions and control rules of the FPPC, a genetic algorithm has been used to construct a FPPC. However, this selftuning approach is time-consuming in obtaining a near optimal solution. In this paper, an easy tuning fuzzy pulse pump controller (ET-FPPC) is proposed for solving the above mentioned problem. ET-FPPC is designed and constructed by using the $\alpha$-cut based Adjustable Defuzzification Method ( $\alpha$-ADM). Only one parameter needs to be tuned in the ETFPPC without tuning the membership functions and control rules. Finally, simulation and experiment are conducted and their results are very close to the theoretical prediction. In comparison with the fuzzy pulse pump controller and the adaptive digital pump controller, the acquisition time of the PFLS is reduced by $45 \%$ and $57 \%$, respectively.
\end{abstract}

\section{Introduction}

The phase-locked servo system (PLS) was first proposed in motor servo control in the 1970's [1] and its digital approach was presented in 1987 [2]. Since then, many digital-pump controllers have been presented in succession to reduce the position acquisition time in PLS [3,5]. Recently, an all-digital controller for PLS was developed to increase the position lock accuracy [6]. In order to raise the acquisition time in the locking process, a fuzzy logic controller was presented for the drives of the PLS [7]. To improve the performance of the phase/frequencylocked servo system (PFLS), we proposed a fuzzy pulse pump controller (FPPC) [9]. The FPPC can provide low overshoot, fast locking response, and short acquisition time for position lock. However, a problem exists in obtaining optimal parameters. A self-turning approach to constructing FPPC using a genetic algorithm called genetic algorithm-based FPPC (GA-FPPC) was proposed to solve the above problem [10]. This approach is useful in obtaining appropriate membership functions and control rules for FPPC. However, this approach is complex and time-consuming in parameter setting. In this paper, we present a new kind of FPPC which can easily, directly and quickly decide on parameters to solve the above mentioned problems.

All of the adaptive fuzzy logic controllers (FLCs) are implemented through tuning membership functions or fuzzy control rules. It is noteworthy that any other variables in an FLC can be used to change the behavior of the FLC. Akifumi Otsubo et al. [11] proposed a new inference method, called productsum-height method, to tune the height of the singleton membership function used for Zero linguistic term in the consequent part. However, the product-sumheight method is based on Rutherford's control rule table. This means that the product-sum-height method is not suitable for any other control rule tables, especially, for the control rule table with a small number of ZO (zero) linguistic terms. Runkler [12] detailed many different defuzzification methods and discussed how to select an appropriate for desired application. This means that the defuzzification method also has a strong influence on FLC-based control systems in addition to membership functions and fuzzy control rules. Additionally, Yager and Filev $[13,14]$ proposed two adjustable defuzzification methods called BAsic Defuzzification Distributions (BADD) and Semi-Linear DEfuzzification (SLIDE). They showed that adjustable defuzzification can easily modify the relation between the output fuzzy set and crisp output value. This indicates that a new kind of tunable FLC without tuning of the membership functions or fuzzy control rules can be developed successfully using adjustable defuzzification methods. Based on the above points, this paper aims at presenting an ET-FPPC by combining the adjustable defuzzification concept with the $\alpha$-cut method. The $\alpha$-cut is very simple to used to modify a fuzzy set. First, the original output fuzzy set of the ET-FPPC is modified by using an $\alpha$-cut. Second, this modified fuzzy output set is regarded as a possibility distribution and is transformed into a probability density distribution by means of normalization operation. Then, a suitable crisp output value (i.e., defuzzified value) can be obtained by 
calculating the expected value of this probability density distribution. This new defuzzification method is called the $\alpha$-cut based Adjustable Defuzzification Method ( $\alpha$-ADM). Then, $\alpha-A D M$ is substituted for the defuzzifier of an FPPC. Finally, the defuzzified value from the $\alpha$-ADM is sent to the digital pump controller to generate a suitable pump voltage to drive the servo motor. This new kind of pump controller is called an easy tuning fuzzy pulse pump controller. The mathematical model of the ET-FPPC based PFLS (EF-PFLS) is also presented to discuss the stability of the EF-PFLS. Finally, the simulation and experiment are conducted to examine the system performance, which is very close to the theoretical prediction.

\section{System Description}

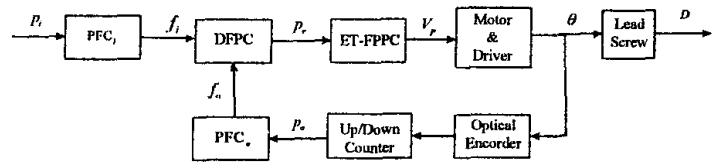

Fig. 1 The block diagram of the EF-PFLS.

The functional scheme of the easy tuning fuzzy pulse pump controller based phase/frequency locked loop servomechanism (EF-PFLS) is shown in Fig. 1. This system is composed of two Position/Frequency Converters (PFCs), a Discrete Frequency/Pulse Comparator (DFPC), an ET-FPPC, a DC motor with a motor driver, an optical encoder, an up/down counter, and a lead screw. The $\mathrm{PFC}_{i}$ and $\mathrm{PFC}_{o}$ are, respectively, used to convert the input pulse number $p_{i}$ (i.e., input position) and output pulse number $p_{o}$ (i.e., feedback position) into the discrete frequencies $f_{i}$ and $f_{o}$. Then, the DFPC compares the discrete frequencies $f_{i}$ and $f_{o}$, and converts the period error $\frac{1}{f_{i}}-\frac{1}{f_{o}}$ into a pulse error $p_{c}$ [6]. After that, the ETFPPC infers a suitable pump voltage $V_{p}$ according to the instant pulse error $p_{e}$ to drive the DC motor. The lead screw is mounted on DC motor to produce linear displacement $D$ from the motor shaft rotation $\theta$. An optical encoder and an up/down counter are also mounted on the shaft of the DC motor to sense and measure the feedback position represented as pulse number $p_{o}$.

\section{Easy Tuning Fuzzy Pulse Pump Controller}

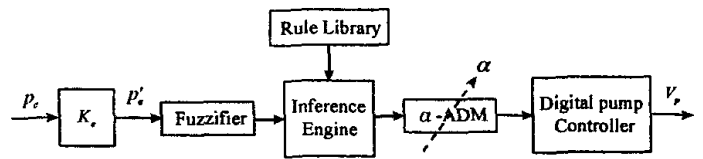

(a)

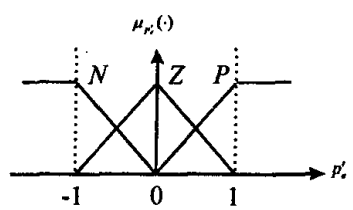

(b)

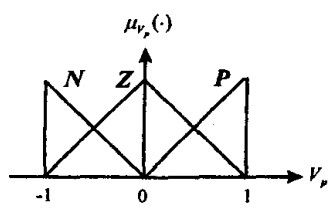

(c)
Fig. 2. (a) The block diagram of the ET-FPPC.

(b) The membership functions of the input fuzzy variable $p_{e}$ for the ET-FPPC.

(c) The membership functions of the output fuzzy variable $V_{p}$ for the ET-FPPC.

The ET-FPPC, shown in Fig. 2(a), contains a digital pump controller, a fuzzifier, a rule library, an inference engine, a normalized factor $K_{\varepsilon}$ and an $\alpha$ ADM. The $\alpha$-ADM is a new kind of adjustable defuzzification method constructed by $\alpha$ cut method and will be detailed in the next section. It is used to instead of tradition defuzzifier in FLC. ET-FPPC's membership function sets of input and output fuzzy variables are shown in Fig. 2(b) and Fig. 2(c), respectively. There are three linguistic terms in each input and output fuzzy variable: $P, Z$ and $N . P$ means positive, $Z$ means zero and $N$ means negative. The rule library of ET-FPPC contains three inference rules. They are:

$$
\begin{aligned}
& \text { IF } p_{e}=\mathrm{P} \text { THEN } V_{p}=\mathrm{P} \\
& \text { IF } p_{e}=\mathrm{Z} \text { THEN } V_{p}=\mathrm{Z} \\
& \text { IF } p_{e}=\mathrm{N} \text { THEN } V_{p}=\mathrm{N}
\end{aligned}
$$

In ET-FPPC, the fuzzifier is used to transfer the input crisp values into fuzzy sets for the inference engine. The inference engine then infers the input fuzzy variable with the rule library and provides a suitable fuzzy set before the $\alpha$-ADM is run. A crisp output value is then obtained from the $\alpha-\mathrm{ADM}$. Finally, the digital pump controller receives the crisp output value and converts it into the pump voltage. Specially, the crisp output value (i.e., pump voltage) can be tuned by using the $\alpha$-ADM. In other words, the pump voltage trajectory can be tuned by $\alpha$ value. In this system, the minimum ( $\mathrm{min}$ ) is used as the intersection operation, and the summation (sum) is used as the union operation.

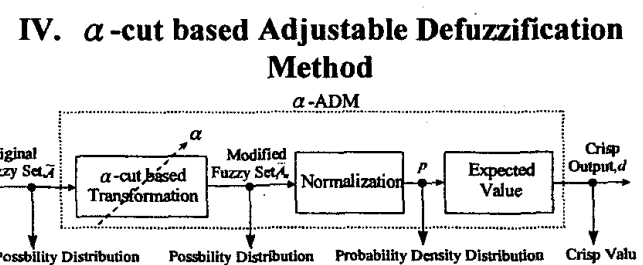

(a) 


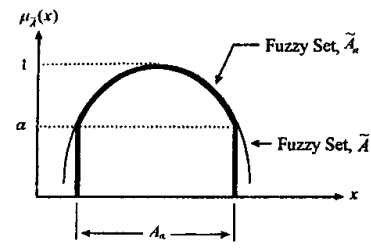

(b)

Fig. 3. (a) The block diagram of the $\alpha-A D M$.

(b) Decomposition of a fuzzy set $\tilde{A}$.

Yager and Filev [13,14] proposed a defuzzification procedure to transform the possibility distribution into a probability density distribution, and the defuzzified value is the expected value of the probability density distribution. In this paper, we use this concept to build the $\alpha$-ADM. The $\alpha$-ADM can be divided into three procedures, as shown in Fig. 3(a). The first procedure of $\alpha-\mathrm{ADM}$ is used to transform the original fuzzy set $\tilde{A}$ into a modified fuzzy set $\tilde{A}_{\alpha}$. The procedure can be written as:

$T_{\alpha}: \widetilde{A} \rightarrow \widetilde{A}_{\alpha}$

where $T_{\alpha}$ is the transformation, which will be discussed later, $\widetilde{A}$ is the original fuzzy set, $\widetilde{A}_{a}$ is the modified fuzzy set.

For clearly explain the $\alpha$-cut based transformation, we assume a convex fuzzy set $\tilde{A}$ defined in the universe of discourse $U$, whose membership function is $\mu_{\tilde{A}}(x)$ and is shown in Fig. 3(b). An $\alpha$-cut of fuzzy set $\tilde{A}$ is a crisp set $A_{\alpha}$, which contains the elements whose membership degrees are greater than or equal to $\alpha$. That is,

$A_{\alpha}=\left\{x \in X \mid \mu_{\tilde{A}}(x) \geq \alpha\right\}, \alpha \in(0,1]$

The characteristic function $\mu_{A_{a}}(x)$ of the crisp set $A_{\alpha}$ is

$\mu_{A_{u}}(x)= \begin{cases}1 & x \in A_{\alpha} \\ 0 & x \notin A_{\alpha}\end{cases}$

Now, we can describe the modified fuzzy set $\widetilde{A}_{\alpha}$ in the same discourse $U$ as follow:

$$
\begin{aligned}
\mu_{\tilde{A}_{\alpha}} & =\left\{\begin{array}{cl}
\mu_{\tilde{A}}(x) & x \in A_{\alpha} \\
0 & x \notin A_{\alpha}
\end{array}\right. \\
& =\left\{\begin{array}{cl}
\mu_{\tilde{A}}(x) & \mu_{\tilde{A}}(x) \geq \alpha \\
0 & \mu_{\tilde{A}}(x)<\alpha
\end{array}\right.
\end{aligned}
$$

Clearly, different $\alpha$ values will result in different modified fuzzy sets $\tilde{A}_{a}$. That is to say, a fuzzy output set of FLC can be modified using $\alpha$-cut based transformation by tuning the $\alpha$ value.

The second procedure of $\alpha$-ADM normalizes the $\mu_{\tilde{A}_{a}}(x)$. This procedure can be written as:

$N(x)=\frac{\mu_{\tilde{A}_{a}}(x)}{\sum_{x \in A_{a}} \mu_{\tilde{A}_{a}}(x)}$

where $N(x)$ is the function of normalized $\mu_{\tilde{A}_{\boldsymbol{a}}}(x)$.

Since

$$
\begin{aligned}
& \text { 1) } N(x) \geq 0 \quad \text { for } x \in A_{\alpha} . \\
& \text { 2) } \sum_{x \in A_{a}} N(x)=1
\end{aligned}
$$

$N(x)$ have the characteristics of a probability density distribution and can be regarded as the probability density function (p.d.f). Thus, the second procedure of the $\alpha$-ADM can be viewed as transformation of the possibility distribution $\mu_{\tilde{A}_{\alpha}}(x)$ into a probability density distribution $N(x)$.

The final procedure in the $\alpha$-ADM is used to determine the crisp output value. Here, the expected value is used to do this. That is,

$$
d=\sum_{x \in A_{\alpha}} N(x) \cdot x
$$

where $d$ is the expected value.

Substituting equation (5) for $N(x)$ in equation (8), we get the defuzzification formula

$d^{\alpha-A D M}=\frac{\sum_{x \in A_{\alpha}} \mu_{\tilde{A}_{\alpha}}(x) \cdot x}{\sum_{x \in A_{\alpha}} \mu_{\tilde{A}_{\alpha}}(x)}$

where $d^{\alpha-A D M}$ is the defuzzified value using the $\alpha$ ADM.

\section{Mathematical Analysis}

The crisp output values of ET-FPPC with different $\alpha$ values are shown in Fig. 4(a). We can find that a larger $\alpha$ value will lead to a larger crisp output value when the pulse error $p_{e}$ is large, and that the crisp output values with different $\alpha$ values are similar when the pulse error $p_{e}$ is close to zero. This is why different $\alpha$ values can result in different step responses in this EF-PFLS. For discussing the stability of the EF-PFLS, the ET-FPPC in the EF- 
PFLS is regarded as an adaptive proportional controller. The adaptive proportional gain $K_{p}$ of the ET-FPPC can be obtained by dividing the output pump voltage $V_{p}$ by the pulse error $p_{e}$. These adaptive proportional gain curves with different $\alpha$ values are shown in Fig. 4(b)

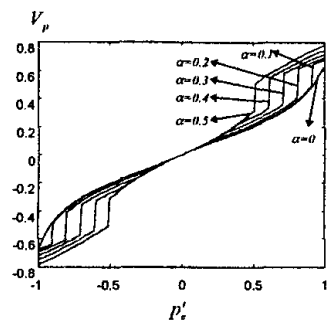

(a)

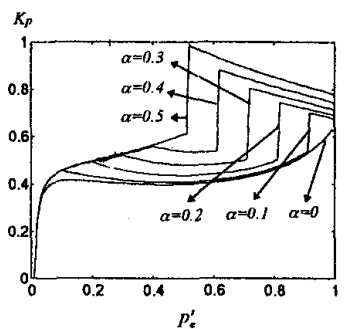

(b)
Fig. 4. (a) The output pump voltage $V_{p}$ of the ETFPPC for different $\alpha$ value.

(b) The adjustable proportional gain $K_{p}$ of the ET-FPPC for different $\alpha$ value.

From Fig. 4(b), we can find that $K_{p}$ can be regarded as an uncertainty parameter with bounded $\left[K_{p}^{-}, K_{p}^{+}\right]$. The maximum value of $K_{p}$ is not larger than 1 (i.e., $K_{p}^{+}=1$ ), and the minimum value of $K_{p}$ is zero (i.e., $K_{p}^{-}=0$ ). Therefore, we can conclude that the range of the adaptive proportional gain $K_{p}$ is $[0,1]$ for any errors with any $\alpha$ values.

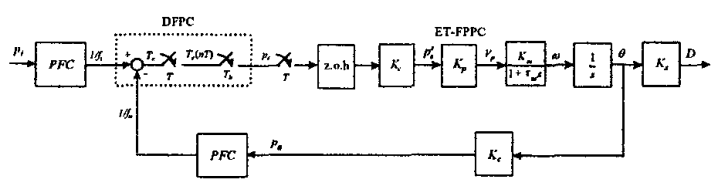

(a)

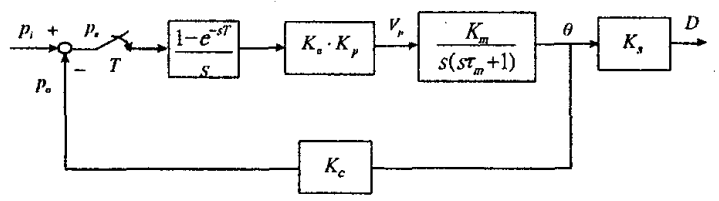

(b)

Fig. 5. (a) The complete mathematical model of the EF-PFLS in locked-in range.

(b) The simplified mathematical model of the EF-PFLS in locked-in range.

Fig. 5 shows the complete and simplified mathematical models of the EF-PFLS in locked-in range, where the relevant parameters are defined as follows: $\tau_{m}$ the mechanical time constant of the motor.

$K_{m}$ the DC gain of the motor with the motor driver $(\mathrm{rad} / \mathrm{sec} / \mathrm{v})$.

$K_{p}$ the adaptive proportional gain of the ET-FPPC.

$K_{s}$ the conversion ratio of the angular displacement to the length of lead screw $(\mathrm{cm} / \mathrm{rad})$.

$K_{e}$ the normalized factor of the input of FPPC.

$K_{c}$ the conversion ratio of the angular displacement to the pulse number $(1 / \mathrm{rad})$.

$T$ the sampling period.

From the Fig 5(b), we have the forward gain

$G(s)=\frac{\theta(s)}{p_{e}(s)}=\frac{K_{e} K_{p} K_{m}\left(1-e^{-s T}\right)}{s^{2}\left(s \tau_{m}+1\right)}$.

With the $z$-transform, we can obtain

$G(z)=\frac{\theta(z)}{p_{e}(z)}=\frac{K_{e} K_{m} K_{p}\left[\left(T-\tau_{m}\left(1-e^{\frac{-T}{\tau_{m}}}\right) z+\tau_{m}\left(1-e^{\frac{-T}{\tau_{m}}}\right)-T e^{\frac{-T}{\tau_{m}}}\right)\right]}{z^{2}-\left(1+e^{\frac{-T}{\tau_{m}}}\right) z+e^{\frac{-T}{\tau_{m}}}}$

The loop gain is

$G H(s)=\frac{K_{c} K_{p} K_{m} K_{c}\left(1-e^{-s T}\right)}{s^{2}\left(s \tau_{m}+1\right)}$

With the $z$-transform, (13) can be transferred to

$$
G H(z)=\frac{K_{e} K_{m} K_{p} K_{c}\left[\left(T-\tau_{m}\left(1-e^{\frac{-T}{\tau_{m}}}\right) z+\tau_{m}\left(1-e^{\frac{-T}{\tau_{m}}}\right)-T e^{\frac{-T}{\tau_{m}}}\right)\right]}{z^{2}-\left(1+e^{\frac{-T}{\tau_{m}}}\right) z+e^{\frac{-T}{\tau_{m}}}} .
$$

From (12) and (14), the closed-loop transfer function can be written as

$$
\frac{\theta(z)}{p_{i}(z)}=\frac{G(z)}{1+G H(z)}=\frac{b_{1} z+b_{0}}{z^{2}+a_{1} z+a_{0}} .
$$

where

$$
\begin{aligned}
& a_{1}=K_{e} K_{m} K_{c} K_{p}\left[T-\tau_{m}\left(1-e^{\frac{-T}{\tau_{m}}}\right)\right]-e^{\frac{-T}{\tau_{m}}}-1 \\
& a_{0}=K_{c} K_{m} K_{c} K_{p}\left[\tau_{m}\left(1-e^{\frac{-T}{\tau_{m}}}\right)-T e^{\frac{-T}{\tau_{m}}}\right]+e^{\frac{-T}{\tau_{m}}} \\
& b_{1}=K_{c} K_{p} K_{m}\left[T-\tau_{m}\left(1-e^{\frac{-T}{\tau_{m}}}\right)\right]
\end{aligned}
$$




$$
b_{0}=K_{e} K_{p} K_{m}\left[\tau_{m}\left(1-e^{\frac{-T}{\tau_{m}}}\right)-T e^{\frac{-T}{\tau_{m}}}\right]
$$

Thus, the system transfer function will be

$$
\frac{D(z)}{p_{i}(z)}=\frac{K_{s}\left(b_{1} z+b_{0}\right)}{z^{2}+a_{1} z+a_{0}} \text {. }
$$

According to the system transfer function, we can have the characteristic equation written as

$$
z^{2}+a_{1} z+a_{0}=0 .
$$

By characteristic equation and Jury's stability test [15], we can have three inequations as follows:

$$
\begin{aligned}
& 1+a_{1}+a_{0}>0 \\
& 1-a_{1}+a_{0}>0 \\
& \left|a_{0}\right|<1
\end{aligned}
$$

These inequations are the necessary and sufficient conditions for system stability. For a given motor with motor driver, optical encoder and lead screw, these values $\tau_{m}, K_{m}, K_{c}$ and $K_{s}$ are known. Therefore, we can compute these inequations (22-24) as functions of sampling period $T$ when $K_{p}$ is determined. Using the above inequations, we can determine sampling period $T$ below which the system is stable.

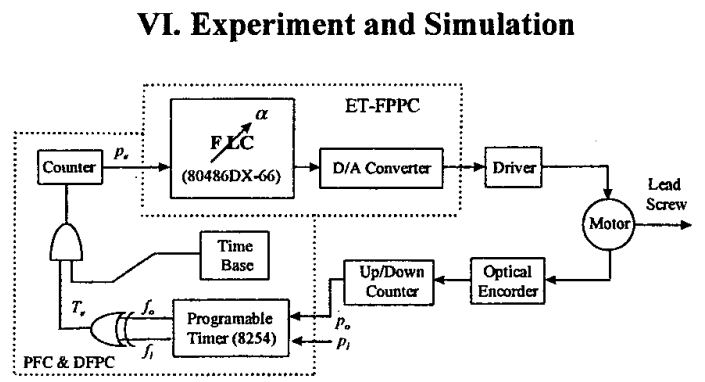

Fig. 6 The block diagram for realizing the EF-PFLS.

The block diagram for realizing the EF-PFLS is shown in Fig. 6. In this design example, the locked-in range is defined in $\left|p_{c}\right| \leq 1000$, the mechanical time constant $\tau_{m}$ of the motor is $115 \mathrm{msec}$, the $D C$ gain $K_{m}$ of the motor with a motor driver is $32.7 \mathrm{~cm} / \mathrm{sec} / V$. Since the pitch of the lead screw is $1 \mathrm{~cm}$ and the pulse number per round of the optical encoder is 1000 , the normalized factor $K_{e}$ is 0.001 , the conversion ratio of the angular displacement to the pulse number $K_{\mathcal{C}}$ is $500 / \pi$ and the conversion ratio of the angular displacement to the length of lead screw $K_{s}$ is $0.5 / \pi$. We can now explore the stability boundary of the sampling period $T$ when $K_{p}$ is equal to 0.5 . The stable range of the sampling period can be obtained by plotting inequations (22-24) in Fig. 7(a). It is easy to see that the maximum stable sampling period $T$ required for this case to be stable is about $0.72 \mathrm{sec}$. Since different $K_{p}$ values result in different maximum stable sampling periods $T$, the distribution of $T$ versus $K_{p}$ is plotted in Fig. 7(b). In this system, the maximum sampling period is $0.45 \mathrm{sec}$ since $K_{p}$ ranges between 0 and 1 .

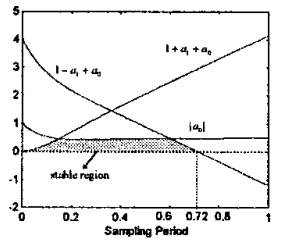

(a)

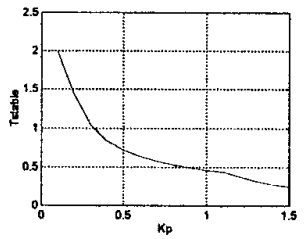

(b)
Fig. 7 (a) The stability boundary of the sampling period $\left(K_{p}=0.5\right)$.

(b) The distribution of the maximum stable sampling period $T$ versus the proportional gain $K_{p}$.

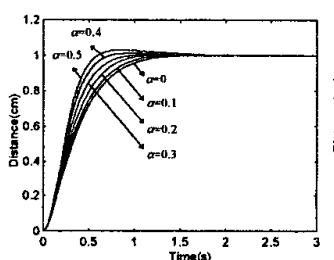

(a)

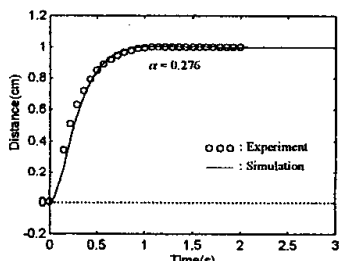

(b)

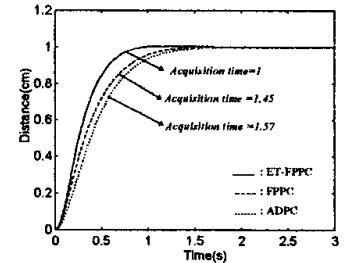

(c)

Fig. 8. (a) Simulated step responses with different $\alpha$ values.

(b) Simulation ( - ) and experimental (ooo) step responses for $\alpha=0.276$.

(c) Step responses of the PFLS with different controllers, which are ET-FPPC ( - ), $\operatorname{FPPC}(---)$ and $\operatorname{ADPC}(\cdots)$.

A simulation and experiment for this example are conducted, as shown in Fig. 8. Fig. 8(a) shows 
different step responses with different $\alpha$ values. The result is the same as the theoretical prediction, where different $\alpha$ values result in different step responses. Since overshoot is not permitted in an excellent servomechanism, $\alpha=0.276$ is adopted for getting null overshoot. From Fig. 8(b), we can easily see that the simulation and the experimental results are very close. Finally, two different pump controllers, a fuzzy pulse pump controller (FPPC) [9] and an adaptive digital pump controller (ADPC) [2], are also selected to construct a PFLS for the purpose of performance comparison. Computer simulations of these three controllers are conducted and shown in Fig. 8(c). In comparison with FPPC and ADPC, the acquisition time of the ET-FPPC is reduced by $45 \%$ and $57 \%$, respectively.

\section{Conclusion}

In this paper, an $\alpha$-cut-based adjustable defuzzification method ( $\alpha-\mathrm{ADM}$ ) is proposed, which can easily change the relation between the crisp output value (i.e., the defuzzified value) and the fuzzy output set by tuning the $\alpha$ value. This property shows that the $\alpha$-ADM can efficiently tune the step response of an FLC-based servo control system. Based on the $\alpha$-ADM, an Easy Tuning Fuzzy Pulse Pump Controller (ET-FPPC) has been designed and realized to achieve an ET-FPPC based Phase/Frequency Locked Loop Servomechanism (EFPFLS). A simulation and experiment are conducted to examine the system performance, which is very close to the theoretical prediction. In comparison with the FPPC and the Adaptive Digital Pump Controller, the acquisition time is reduced by $45 \%$ and $57 \%$, respectively.

\section{References:}

[1] A. W. Moore, "Phase locked loops for motorspeed control," IEEE Spectrum, pp.61-67, Apr. 1973.

[2] G. C. Hsieh, P. P. Wu, C. H. Lee, and C. H. Liu, "An adaptive digital pump controller for phaselocked servo systems," IEEE Trans. Ind. Electron., vol. IE-34, no. 3, pp. 379-386, Aug. 1987.

[3] G. C. Hsieh, "A study on position servo control systems by frequency-locked servo system using the slope-varied digital-pump technique," IEEE Trans. Ind. Electron., vol. 36, no. 3, pp. 365-373, Aug. 1989.

[4] J. C. Li, G. C. Hsieh, "A phase/frequencylocked controller for stepping servo control system," IEEE Trans. Ind. Electron., vol. 39, no.
2, pp.112-119, Apr. 1992.

[5] M. F. Lai, G. C. Hsieh, Y. P. Wu "Variable slope pulse pump controller for stepping position servo control system using frequencylocked technique," IEEE Trans. Ind. Electron., vol. 42, no. 3, pp. 290-299, June 1995.

[6] G. C. Hsieh, L. R. Chen, K. L. Hsieh, H. C. Wu, and J. H. Hsu, "Adaptive pulse-pump controller for X-Y mode frequency-locked stepping servomechanism," $J$. of the Chinese Institute of Eng., vol. 21, no. 1, pp. 47-58, 1998.

[7] M. F. Lai, M. Nakano, and G. C. Hsieh, "Application of fuzzy logic in phase-locked loop speed control of induction motor drive," IEEE Trans. Ind. Electron., vol. 43, no. 6, pp. 630-639, Dec. 1996.

[8] G. C. Hsieh, and J. C. Hung, "Phase-locked loop techniques-A survey," IEEE Trans. Ind. Electron., vol. 43, no. 6, pp. 609-615, Dec. 1996.

[9] G. C. Hsieh, H. M. Lee, L. R. Chen and Y. J. Penth, "A study of phase/frequency-locked servo system by fuzzy control technique," in Proc. IEEE Int. Conf. SMC., 1997, pp. 13231328.

[10] G. C. Hsieh, H. M. Lee, L. R. Chen and Y. J. Penth, "Genetic Algorithm-Based Fuzzy PulsePump Controller for Phase/Frequency-Locked Servomechanism," in Proc. IEEE IECON., 1998, pp. 81-87.

[11] Akifumi Otsubo, Kenichiro Hayashi, Shuta Murakami, and Mikio Maeda, "Improvement of control performance for low-dimensional number of fuzzy labeling using simplified inference method," Fuzzy Sets and Systems, pp. 37-44, 1997.

[12] T. A. Runkler, "Selection of appropriate defuzzification methods using application specific properties," IEEE Trans. Fuzzy Systems, vol. 5, no. 1, pp. 72-79, Feb. 1997.

[13] Dimitar P. Filev and Ronald R. Yager, "A generalized defuzification method via Bad distributions," Int. J. Intelligent Systems, vol. 6, pp. 687-697, 1991.

[14] Ronald R. Yager and Dimitar P. Filev, " SLIDE: a simple adaptive defuzzification method," IEEE Trans. Fuzzy systems, vol. 1, no. 1, pp. 6978, Feb, 1993.

[15] B. C. Kuo, Digital control system, $2^{\text {nd }}$ edition, saundars college, 1992

[16] A. Dan-Isa, and D. P. Atherton, "Time-domain optimal method for the design of optimal liner controllers," IEE Proc. Control Theory Appl., vol. 144, no. 4, pp. 287-292, July 1997. 